\title{
ON THE RADIUS AND THE RELATION BETWEEN THE TOTAL GRAPH OF A COMMUTATIVE RING AND ITS EXTENSIONS
}

\section{Zoran Pucanović and Zoran Petrović}

Communicated by Žarko Mijajlović

\begin{abstract}
We discuss the determination of the radius of the total graph of a commutative ring $R$ in the case when this graph is connected. Typical extensions such as polynomial rings, formal power series, idealization of the $R$-module $M$ and relations between the total graph of the $\operatorname{ring} R$ and its extensions are also dealt with.
\end{abstract}

\section{Introduction}

Every ring $R$ in this paper is assumed to be commutative with 1 . Let $Z(R)$ denote the set of its zero-divisors, $Z(R)^{*}=Z(R) \backslash\{0\}$ the set of its non-zero zero-divisors, $\operatorname{Reg}(R)=R \backslash Z(R)$ its set of regular elements and $\operatorname{Nil}(R)$ the ideal of its nilpotent elements. Let $R[x], R[[x]]$ and $R(+) M$, where $M$ is an $R$-module, represent standard notation for the polynomial ring, formal power series ring and idealization of the module $M$. The set of annihilators of elements of an $R$-module $M$ is denoted by $Z(M)$. Basic definitions and results concerning commutative rings may be found in any standard textbook on commutative algebra, e.g. 14.

The idea to associate a graph to a commutative ring, where all elements of the ring are vertices of that graph, first appears in 7 . which deals with graph coloring. In [2], Anderson and Livingstone take the nonzero zero-divisors for the vertices of the graph, and two vertices $x, y \in Z(R)^{*}$ are adjacent iff $x y=0$. The resulting graph $\Gamma(R)$ is the zero-divisor graph of the ring $R$. The authors have, among other results, proved that this graph is always connected and that $\operatorname{diam} \Gamma(R) \leqslant 3[\mathbf{2}$, Theorem 2.3]. Papers [2, 3, 15, 5, 6, 16, 17] deal with various properties of this graph.

2010 Mathematics Subject Classification: Primary 13A99; Secondary 05C25.

Key words and phrases: Commutative rings; zero-divisors; total graph.

The second author is partially supported by Ministry of Science and Environmental Protection of Republic of Serbia Project \#174032. 
In 1 Anderson and Badawi define, for a commutative ring $R$ with 1 , its total graph $T(\Gamma(R))$. The set of vertices of this graph is $R$ and two different elements $x, y \in R$ are adjacent iff $x+y \in Z(R)$. The rich and complex structure of this graph makes it an interesting object for study. For example, one may, for any $n \in \mathbb{N}$, construct a ring $R_{n}$ such that the corresponding graph has diameter $n[\mathbf{1}$, Example 3.8]; this is rather different than the case of zero-divisor graphs. Unlike $\Gamma(R)$, the graph $T(\Gamma(R))$ is connected only under certain conditions. The properties of this graph naturally depend on the fact whether $Z(R)$ is an ideal of $R$ or not; so there are two separate cases to discuss. Nevertheless, in both cases, the total graph contains induced subgraphs $\operatorname{Reg}(\Gamma(R)), \mathrm{Z}(\Gamma(R))$ and $\operatorname{Nil}(\Gamma(R))$ whose vertices lie in $\operatorname{Reg}(R), Z(R)$ and $\operatorname{Nil}(R)$. These subgraphs add to the understanding of the total graph itself.

Vertices $x$ and $y$ of the graph $\Gamma$ are connected if there is a path in $\Gamma$ beginning at one of them and ending at the other. If every vertex is connected to every other vertex, the graph $\Gamma$ is connected. For two different vertices $x, y \in V(\Gamma)$, one defines the distance $d(x, y)$ as the length of the shortest path between $x$ and $y$ if the vertices in question are connected, and one puts $d(x, y)=\infty$ in case they are not. The diameter of the graph $\Gamma$ is $\operatorname{diam}(\Gamma)=\sup \{d(x, y) \mid x, y \in \Gamma\}$, and the eccentricity of the vertex $x$ is the distance between $x$ and the vertex which is at the greatest distance from $x, e(x)=\max \{d(x, y) \mid y \in \Gamma\}$. The radius of the graph $\Gamma$, $r(\Gamma)$, is defined by $r(\Gamma)=\min \{e(x) \mid x \in \Gamma\}$, and the center of the graph is the set of all of its vertices whose eccentricity is minimal, i.e., it is equal to the radius. So, the radius of the graph is equal to the smallest eccentricity and diameter to the largest eccentricity of a vertex in this graph. It is well known that for connected graphs of diameter $d$ and radius $r$, one has $r \leqslant d \leqslant 2 r$.

\section{Radius of the total graph of a commutative ring}

2.1. The set of zero-divisors $Z(R)$ is an ideal of $R$. Since $Z(R)$ is always a union of prime ideals of the ring $R$ [14, if $Z(R)$ is an ideal, it has to be a prime ideal. Note that in this case the induced subgraph $\mathrm{Z}(\Gamma(R))$ is complete; so $r(\mathrm{Z}(\Gamma(R)))=\operatorname{diam}(\mathrm{Z}(\Gamma(R)))=1$. However, in this case the total graph $T(\Gamma(R))$ is not connected since no vertex from $Z(R)$ is adjacent to a vertex from $\operatorname{Reg}(R)$. Therefore, it does not make sense to discuss a radius of $T(\Gamma(R))$, but one may check the radius of its subgraph of regular elements. The structure of this graph is given in [1, Theorem 2.2]. A corollary to this theorem is the following result [1, Theorem 2.4]: $\operatorname{Reg}(\Gamma(R))$ is connected if and only if $R / Z(R) \cong \mathbb{Z}_{2}$ or $R / Z(R) \cong \mathbb{Z}_{3}$. In the first case, $\operatorname{Reg}(\Gamma(R))$ is the complete graph $K^{\alpha}$, where $\alpha=|Z(R)|$; therefore

$$
r(\operatorname{Reg}(\Gamma(R)))=\operatorname{diam}(\operatorname{Reg}(\Gamma(R)))=1 .
$$

In the second case, $\operatorname{Reg}(\Gamma(R))$ is the complete bipartite graph $K^{\alpha, \alpha}$; consequently

$$
r(\operatorname{Reg}(\Gamma(R)))=\operatorname{diam}(\operatorname{Reg}(\Gamma(R)))=2 .
$$

2.2. The set of zero-divisors $Z(R)$ is NOT an ideal of $R$. When the zerodivisors do not form an ideal, the structure of the total graph $T(\Gamma(R))$ can not be completely determined as in the previous case. Namely, the subgraphs $\mathrm{Z}(\Gamma(R))$ 
and $\operatorname{Reg}(\Gamma(R))$ are not separated from each other since there exist $x, y \in Z(R)$ such that $x+y \notin Z(R)$; therefore the vertices $-x \in Z(R)$ and $x+y \in \operatorname{Reg}(R)$ are adjacent. Note that $|Z(R)| \geqslant 3$. Since in this case there exist rings whose total graphs have arbitrary large diameter, it is natural to ask what happens to the radius of the total graph. The answer is somewhat unexpected, namely we show that the radius is always equal to the diameter. As a motivating example, we may consider a finite commutative ring $R$ such that $Z(R)$ is not an ideal of $R$. The diameter of the total graph of such a ring is always 2 [1 Theorem 3.4].

THEOREM 2.1. Let $R$ be a finite commutative ring with 1 such that $Z(R)$ is not an ideal of $R$. Then $r(T(\Gamma(R)))=2$.

Proof. Since $d=\operatorname{diam}(T(\Gamma(R)))=2$, one has $r=r(T(\Gamma(R))) \leqslant 2$; so we only have to show that $r \neq 1$. Assume that $r=1$. In this case there exists $x \in R$ such that $e(x)=1$, i.e., $x$ is adjacent to every other vertex. It follows that $x \in Z(R)$ ( $x$ is adjacent to 0 ); moreover $x \neq 0$ (otherwise 1 is adjacent to 0 and it would follow that $R$ is the zero ring). Since $Z(R)$ is not an ideal, there are $a, b \in Z(R)$ such that $a+b \in \operatorname{Reg}(R)$. So, $x \neq a, x \neq b$ and $x \neq a+b$. The vertex $c=-x+a+b$ does not belong to $\{a, b, 0\}$ and it is adjacent to $x$. We conclude that $a+b \in Z(R)$ which is impossible. Therefore, the radius must be 2 .

Suppose that $R$ is an arbitrary commutative ring with 1 such that $Z(R)$ is not an ideal of $R$. By [1, Theorem 3.3], $T(\Gamma(R))$, is connected if and only if $R$ is generated by zero-divisors, $\langle Z(R)\rangle=R$, i.e., $R=\left\langle z_{1}, z_{2}, \ldots, z_{n}\right\rangle$ for some $z_{1}, z_{2}, \ldots, z_{n} \in Z(R)$. Moreover, if $n \geqslant 2$ is the minimal number of zero-divisors which generate $R$, then $\operatorname{diam}(T(\Gamma(R)))=n=d(0,1)$ [1 Theorem 3.4]. Let us now prove that under these conditions the radius of the total graph is equal to its diameter.

THEOREM 2.2. Let $R$ be a commutative ring with 1 such that $Z(R)$ is not an ideal of $R$, and let $n \geqslant 2$ be the smallest integer such that $R=\left\langle z_{1}, \ldots, z_{n}\right\rangle$, for some $z_{1}, \ldots, z_{n} \in Z(R)$. Then $r(T(\Gamma(R)))=n$.

Proof. We know that $\operatorname{diam}(T(\Gamma(R)))=n$; so we only have to prove that $r \leqslant n-1$ is not possible. Assume that $r \leqslant n-1$. So, there exists $x \in R$ such that $e(x) \leqslant n-1$, i.e.,

$$
(\forall y \in R) d(x, y) \leqslant n-1 .
$$

In particular, $d(x, 1+x) \leqslant n-1$ and $d(x, 1-x) \leqslant n-1$. Let

$$
x-s_{1}-s_{2}-\cdots-s_{k-2}-1+(-1)^{k-1} x
$$

be a path of length $k-1 \leqslant n-1$ in $T(\Gamma(R))$. We get $k-1$ zero-divisors

$$
x+s_{1}, s_{1}+s_{2}, \ldots, s_{k-3}+s_{k-2}, s_{k-2}+1+(-1)^{k-1} x
$$

for which it holds that

$$
\left\langle x+s_{1}, s_{1}+s_{2}, \ldots, s_{k-3}+s_{k-2}, s_{k-2}+1+(-1)^{k-1} x\right\rangle \subseteq\langle Z(R)\rangle=R .
$$

Since

$$
1 \in\left\langle x+s_{1}, s_{1}+s_{2}, \ldots, s_{k-3}+s_{k-2}, s_{k-2}+1+(-1)^{k-1} x\right\rangle
$$


we must have

$$
\left\langle x+s_{1}, s_{1}+s_{2}, \ldots, s_{k-3}+s_{k-2}, s_{k-2}+1+(-1)^{k-1} x\right\rangle=R .
$$

It follows that $R$ may be generated by $k-1$ zero-divisors, which is a contradiction $(k-1<n)$.

\section{Total graph of some typical ring extensions}

3.1. Polynomial rings. Let $R[x]$ be the polynomial ring over $R$. First of all, $Z(R) \subseteq Z(R[x]) \subseteq Z(R)[x]$ always holds. The second inclusion may be proper, for example, $2+3 x \in Z\left(\mathbb{Z}_{6}\right)[x] \backslash Z\left(\mathbb{Z}_{6}[x]\right)$. It is clear that the first inclusion may be proper as well. The well-known McCoy's theorem gives a desription of the set of zero-divisors in a polynomial ring: $f(x) \in Z(R[x])$ iff there exists $r \in R^{*}$ such that $r f(x)=0$. Therefore, not only the coefficients have to be zero-divisors, but the ideal generated by these coefficients should have a nonzero annihilator. A ring $R$ is a McCoy ring if and only if for every finitely generated ideal $I \subseteq Z(R)$ it is true that $A n n(I) \neq 0$. It is well known that a polynomial $\operatorname{ring} R[x]$ is always a McCoy ring.

The structure of the total graph $T(\Gamma(R[x]))$ of a polynomial ring depends on the fact whether $Z(R[x])$ is an ideal of $R[x]$ or not. According to [15, Theorem 3.3], $Z(R[x])$ is an ideal of $R[x]$ if and only if $R$ is a McCoy ring such that $Z(R)$ is an ideal of $R$. This result allows us to characterize the structure of the total graph of polynomial rings.

Let us first suppose that $Z(R[x])$ is an ideal of $R[x]$. It is evident that in this case the subgraph of zero-divisors $Z(\Gamma(R[x]))$ is complete. One has $Z(R[x])=$ $Z(R)[x]$; consequently

$$
R[x] / Z(R[x])=R[x] / Z(R)[x] \cong(R / Z(R))[x] .
$$

On the right-hand side is a polynomial ring which clearly cannot be isomorphic to $\mathbb{Z}_{2}$ or to $\mathbb{Z}_{3}$ and we conclude that $\operatorname{Reg}(\Gamma(R[x]))$ is not connected [1, Theorem 2.4]. From the previous discussion, one can characterize the structure of the total graph of a polynomial ring in which zero-divisors form an ideal.

THEOREM 3.1. Let $R$ be a McCoy ring such that $Z(R)$ is an ideal of $R$. In this case the total graph $T(\Gamma(R[x]))$ is not connected. The induced subgraph $Z(\Gamma(R[x]))$ is complete, while $\operatorname{Reg}(\Gamma(R[x]))$ is not connected as well.

Remark 3.1. Since $R[x]$ is always a McCoy ring and $R[x, y]=R[x][y]$, from the hypothesis that $R$ is a McCoy ring and $Z(R)$ is an ideal of $R$, it follows that $\operatorname{Reg}(\Gamma(R[x, y]))$ is not connected.

Let us now concentrate on the case when $Z(R[x])$ is not an ideal of $R[x]$. We first prove the following useful lemma.

Lemma 3.1. Let $R$ be a ring such that $Z(R)$ is not an ideal of $R$. Then:

$$
\langle Z(R[x])\rangle=R[x] \quad \text { iff } \quad\langle Z(R)\rangle=R .
$$


Proof. Suppose that $\langle Z(R[x])\rangle=R[x]$. Therefore, there exist polynomials $f_{1}(x), \ldots, f_{n}(x) \in Z(R[x])$ such that $f_{1}(x)+\cdots+f_{n}(x)=1$. It follows that $z_{1}+$ $\cdots+z_{n}=1$, where $z_{1}, \ldots, z_{n}$ are constant coefficients of the previous polynomials. Since $Z(R[x]) \subseteq Z(R)[x]$, all coefficients of these polynomials are zero-divisors. Therefore, $z_{1}, \ldots, z_{n} \in Z(R)$ as well. So, $R=\langle Z(R)\rangle=\left\langle z_{1}, \ldots, z_{n}\right\rangle$. The other implication is trivial.

Using this lemma, [1 Theorem 3.3 and Theorem 3.4], as well as Theorem 2.2, we arrive at the following result.

THEOREM 3.2. Let $R$ be a ring such that $Z(R)$ is not an ideal of $R$. Then $T(\Gamma(R[x]))$ is connected if and only if $T(\Gamma(R))$ is connected, i.e., there exist $z_{1}, \ldots, z_{n} \in Z(R)$ such that $R=\langle Z(R)\rangle=\left\langle z_{1}, \ldots, z_{n}\right\rangle$. If $n$ is the minimal number of such generators then

$$
\operatorname{diam} T(\Gamma(R[x]))=r(\Gamma(R[x]))=n .
$$

3.2. Rings of formal power series. Although rings of formal power series share some properties with polynomial rings, they do differ when it comes to zerodivisors. For example, McCoy's theorem does not hold for $R[[x]]$. In [10, Fields has presented an example of formal power series, with an invertible coefficient, which is nonetheless proper zero-divisor. This example shows that $Z(R[[x]]) \subseteq Z(R)[[x]]$ need not hold. It is clear that the reverse inclusion may not hold. The reason for this lies in the nilpotent elements. Namely, if the ring $R$ is reduced, it has been shown in the paper [11 that $f(x) \in Z(R[[x]])$ if and only if there exists $z \in Z(R)^{*}$ such that $z f(x)=0$. The papers [5, 15] deal with the problem of determining the diameter of the graphs $\Gamma(R[x])$ and $\Gamma(R[[x]])$. The authors have presented the complete result for diam $\Gamma(R[x])$ for an arbitrary ring $R$, and $\operatorname{diam} \Gamma(R[[x]])$ when a ring $R$ is reduced. For nonreduced rings the problem of determining this diameter remains open.

We now concentrate on the case of a reduced ring, and we analyze the total graph $T(\Gamma(R[[x]]))$. The obvious question one might ask is whether $Z(R[[x]])$ is an ideal of $R[[x]]$. Let us suppose that $Z(R)$ is an ideal of $R$. It is clear that $Z(R[[x]]) \subseteq Z(R)[[x]]$. The equality in this case holds if and only if the ring $R$ is a countably McCoy ring, i.e., if every countably generated ideal $I \subseteq Z(R)$ has a nonzero annihilator. We present this as the following lemma.

Lemma 3.2. Let $R$ be a reduced, countably McCoy ring such that $Z(R)$ is an ideal of $R$. Then $Z(R[[x]])$ is an ideal of $R[[x]]$ and $Z(R[[x]])=Z(R)[[x]]$.

Under these conditions

$$
R[[x]] / Z(R[[x]])=R[[x]] / Z(R)[[x]] \cong(R / Z(R))[[x]] .
$$

The right-hand side is not isomorphic to $\mathbb{Z}_{2}$ or to $\mathbb{Z}_{3}$, so one has the result analogous to the one for polynomial rings.

THEOREM 3.3. Let $R$ be a reduced, countably McCoy ring such that $Z(R)$ is an ideal of $R$. Then the total graph $T(\Gamma(R[[x]]))$ is not connected, the induced subgraph $Z(\Gamma(R[[x]]))$ is complete and $\operatorname{Reg}(\Gamma(R[[x]]))$ is not connected. 
Using the same reasoning as in the polynomial case one can prove that for the reduced ring $R$ such that $Z(R)$ is an ideal of $R$ one has

$$
\langle Z(R[[x]])\rangle=R[[x]] \quad \text { iff } \quad\langle Z(R)\rangle=R .
$$

So, we have the following theorem.

THEOREM 3.4. Let $R$ be a reduced ring such that $Z(R)$ is not an ideal of $R$. Then $T(\Gamma(R[[x]]))$ is connected if and only if $T(\Gamma(R))$ is connected, i.e., there exist $z_{1}, \ldots, z_{n} \in Z(R)$ such that $R=\langle Z(R)\rangle=\left\langle z_{1}, \ldots, z_{n}\right\rangle$. If $n \geqslant 2$ is the smallest number of such generators we have

$$
\operatorname{diam} T(\Gamma(R[[x]]))=r(\Gamma(R[[x]]))=n .
$$

3.3. Idealization. The idealization method is rather important for constructions of rings with zero-divisors. Let $R$ be a commutative ring and $M$ an $R$-module. Operations on $R \times M$ are defined as follows: $\left(r_{1}, m_{1}\right)+\left(r_{2}, m_{2}\right)=\left(r_{1}+r_{2}, m_{1}+m_{2}\right)$ and $\left(r_{1}, m_{1}\right)\left(r_{2}, m_{2}\right)=\left(r_{1} r_{2}, r_{1} m_{2}+r_{2} m_{1}\right)$. The commutative ring obtained using this construction is called the idealization of a module $M$ and is denoted by $R(+) M$. The module $M$ can now be seen as the ideal $0(+) M$ of the ring $R(+) M$. Since $\left(0, m_{1}\right)\left(0, m_{2}\right)=(0,0)$ this ideal is nilpotent of index 2 . Note also that, by identifying $m \in M$ with $(0, m) \in R(+) M$, all elements of the module $M$ are zerodivisors in the idealization. Different aspects of the idealization are thoroughly investigated in [4, while the papers [6, 3] deal with $\Gamma(R(+) M)$. We consider the total graph $T(\Gamma(R(+) M))$. Zero-divisors in the idealization are given by (see $\mathbf{1 3}$, Theorem 25.3]): $Z(R(+) M)=\{(r, m) \mid r \in Z(R) \cup Z(M), m \in M\}$. Let us first discuss a few motivating examples.

ExAmple 3.1. The idealization $R(+) R$ of a module $R$ for an arbitrary commutative ring $R$.

We prove that the properties of the graph of the ring and its idealization remain the same.

Let us first assume that $Z(R)$ is an ideal of $R$. We know that $T(\Gamma(R))$ is not connected, $Z(\Gamma(R))$ is complete, while $\operatorname{Reg}(\Gamma(R))$ is connected if and only if $R / Z(R)$ is isomorphic to $\mathbb{Z}_{2}$ or to $\mathbb{Z}_{3}$. Since $Z(R)$ is an ideal of $R, Z(R(+) R)=Z(R)(+) R$ is an ideal of $R(+) R$. So $T(\Gamma(R(+) R))$ is not connected and $Z(\Gamma(R(+) R))$ is complete. From $(R(+) R) /(Z(R(+) R))=(R(+) R) /(Z(R)(+) R) \cong R / Z(R)(+) 0 \cong$ $R / Z(R)$, it follows that $\operatorname{Reg}(\Gamma(R(+) R))$ is connected if and only if $\operatorname{Reg}(\Gamma(R))$ is connected.

Suppose that $Z(R)$ is not an ideal of $R$. Then $Z(R(+) R)=Z(R)(+) R$ is not an ideal of $R(+) R$. So, $T(\Gamma(R(+) R))$ is connected if and only if $T(\Gamma(R))$ is connected and $\operatorname{diam}(T(\Gamma(R(+) R)))=\operatorname{diam}(T(\Gamma(R))$. This may be proved by comparing the path $x-s_{1}-\cdots-s_{n}-y$ in $T(\Gamma(R))$ with $(x, 0)-\left(s_{1}, t_{1}\right)-\cdots-\left(s_{n}, t_{n}\right)-(y, 0)$ and $(x, a)-\left(s_{1}, 0\right)-\cdots-\left(s_{n}, 0\right)-(y, b)$, which are paths in $T(\Gamma(R(+) R))$, and one can find it in [1, Theorem 3.16].

EXAMPLE 3.2. The idealization $\mathbb{Z}[x] /\left(x^{2}\right)(+) \mathbb{Z}_{10}$. 
We show that the properties of the graphs $T(\Gamma(R))$ and $T(\Gamma(R(+) M))$ substantially differ in this case. The first one is not connected, while the second one is connected.

Let $R=\mathbb{Z}[x] /\left(x^{2}\right)=\{a+b x \mid a, b \in \mathbb{Z}\}, M=\mathbb{Z}_{10}$. It is easy to check that $M$ is an $R$-module if the action is defined by $(a+b x) m=a m$. The set $Z(R)=\{a x \mid a \in \mathbb{Z}\}$ of zero-divisors of the ring $R$ is the (principal) ideal of $R$, so $T(\Gamma(R))$ is not connected and $Z(\Gamma(R))$ is complete. We also have $R / Z(R) \cong \mathbb{Z}$. Therefore, $\operatorname{Reg}(\Gamma(R))$ is not connected.

On the other hand, $Z(M)=P \cup Q$, where $P=2 \mathbb{Z}+(x)$ and $Q=5 \mathbb{Z}+(x)$ are prime ideals, is not an ideal of $R$. Note that $Z(R) \subseteq Z(M)$. It is easy to see that the set of zero-divisors $Z(R(+) M)=\left\{(z, m) \mid z \in P \cup Q, m \in \mathbb{Z}_{10}\right\}$, also fails to be an ideal of $R(+) M$. Let us, for example, take $z_{1}=(2,0), z_{2}=$ $(5,0)$. Then $z_{1}, z_{2} \in Z(R(+) M)$, but $z_{1}+z_{2}=(7,0) \in \operatorname{Reg}(R(+) M)$. Since $(3,5) z_{1}+(-1,0) z_{2}=(1,0)$, we have $\langle Z(R)\rangle=\left\langle z_{1}, z_{2}\right\rangle=R(+) M$, and $T(\Gamma(R(+) M)$ is connected with diameter 2 . The subgraph $Z(\Gamma(R(+) M)$ is also connected with diameter 2 as well. The subgraph $\operatorname{Reg}(\Gamma(R(+) M)$ is actually complete, since for $\left(s_{1}, m_{1}\right),\left(s_{2}, m_{2}\right) \in \operatorname{Reg}\left(\Gamma(R(+) M)\right.$ it holds that $s_{1}+s_{2} \in P$ and therefore $\left(s_{1}+s_{2}\right.$, $\left.m_{1}+m_{2}\right) \in Z(\Gamma(R(+) M)$.

These examples motivate us to check under what conditions the properties of the total graph of the ring $R$ pass onto the total graph of the idealization $R(+) M$. Since clearly $Z(R)(+) M \subseteq Z(R(+) M)$, we first need to check under what conditions the equality holds, as well as the conditions ensuring that this set is an ideal. In the proofs we use the general result concerning ideals in the idealization [4, Theorem 3.1]: for an ideal $I$ of the $\operatorname{ring} R$ and submodule $N$ of the $R$-module $M, I(+) N$ is an ideal of $R(+) M$ if and only if $I M \subseteq N$. Then $(R(+) M) /(I(+) N) \cong(R / I)(+)(M / N)$. We have the following theorem.

TheOREM 3.5. Let $M$ be an $R$-module such that $Z(M) \subseteq Z(R)$. Then the following conditions are equivalent:

(i) $Z(R(+) M)$ is an ideal of $R(+) M$. (ii) $Z(R)$ is an ideal of $R$.

In addition to that $Z(R)(+) M=Z(R(+) M)$.

Proof. Let us first suppose that $Z(R)$ is an ideal of $R$. Since $Z(M) \subseteq Z(R)$, we have $Z(R) \cup Z(M)=Z(R)$ and therefore $Z(R(+) M)=Z(R)(+) M$. The set on the right-hand side is an ideal according to [4, Theorem 3.1].

Suppose that $Z(R(+) M)$ is an ideal of $R(+) M$ and let $z_{1}, z_{2} \in Z(R)$. Then $\left(z_{1}, 0\right),\left(z_{2}, 0\right) \in Z(R(+) M)$; so, $\left(z_{1}+z_{2}, 0\right) \in Z(R(+) M)$. From this we conclude that $z_{1}+z_{2} \in Z(R) \cup Z(M)=Z(R)$. Likewise, if $r \in R$ and $z \in Z(R)$ then $(r, 0) \in$ $R(+) M$ and $(z, 0) \in Z(R(+) M)$. Consequently, $(r, 0)(z, 0)=(r z, 0) \in Z(R(+) M)$, and we have $r z \in Z(R) \cup Z(M)=Z(R)$.

THEOREM 3.6. Let $R$ be a commutative ring such that $Z(R)$ is an ideal and let $M$ be an $R$-module such that $Z(M) \subseteq Z(R)$. Then $T(\Gamma(R(+) M))$ is disconnected, $Z(\Gamma(R(+) M))$ is complete, while $\operatorname{Reg}(\Gamma(R(+) M))$ is connected if and only if $\operatorname{Reg}(\Gamma(R))$ is connected. 
Proof. From the previous theorem and [1, Theorem 2.1], it follows that $T(\Gamma(R(+) M))$ is disconnected and $Z(\Gamma(R(+) M))$ is complete. Furthermore,

$$
(R(+) M) /(Z(R(+) M))=(R(+) M) /(Z(R)(+) M) \cong R / Z(R)(+) 0 \cong R / Z(R) .
$$

Therefore, $\operatorname{Reg}(\Gamma(R(+) M))$ is connected if and only if $\operatorname{Reg}(\Gamma(R))$ is connected.

The case when $Z(R)$ is not an ideal of $R$ has been discussed in [1]. The authors have shown that the connectedness of $T(\Gamma(R))$ implies the connectedness of $T(\Gamma(R(+) M))$ and that $\operatorname{diam}(T(\Gamma(R(+) M))) \leqslant \operatorname{diam}(T(\Gamma(R)))$ [1 Theorem 3.17]. Under the additional assumption $Z(R)(+) M=Z(R(+) M)$, we have that the graph $T(\Gamma(R(+) M))$ is connected if and only if $T(\Gamma(R))$ is connected and $\operatorname{diam}(T(\Gamma(R(+) M)))=\operatorname{diam}(T(\Gamma(R)))$ [1, Theorem 3.16].

3.4. Matrices. Although in this section we venture into the noncommutative algebra, the case of the total graph of the matrix ring $M_{n}(R)$ for an arbitrary commutative ring $R$ is worth mentioning. The zero-divisor graph for a noncommutative ring may be defined in various ways, but we follow [18. For the case of the ring of matrices over commutative rings see [8].

For a ring $R, Z_{L}(R)=\left\{x \in R \mid x a=0\right.$, for some $\left.a \in R^{*}\right\}$ is the set of its left zero-divisors, $Z_{R}(R)=\left\{x \in R \mid b x=0\right.$, for some $\left.b \in R^{*}\right\}$ is the set of its right zero-divisors, while $Z(R)=Z_{L}(R) \cup Z_{R}(R)$ is the set of all zero-divisors in this ring. Redmond defines directed and undirected graphs, $\Gamma(R)$ and $\bar{\Gamma}(R)$. In both cases vertices are nonzero zero-divisors, and $x \rightarrow y$ in $\Gamma(R)$ iff $x y=0$, while $x-y$ in $\bar{\Gamma}(R)$ iff $x y=0$ or $y x=0$. According to [18, Theorem 2.3], the graph $\Gamma(R)$ is connected if and only if $Z_{L}(R)=Z_{R}(R)$ and then $\operatorname{diam}(\Gamma(R)) \leqslant 3$, while $\bar{\Gamma}(R)$ is always connected and $\operatorname{diam}(\bar{\Gamma}(R)) \leqslant 3$, [18, Theorem 3.2].

We define the total (undirected) graph $T(\Gamma(R))$ of a noncommutative ring $R$ in the same way as for the commutative case. The vertices are all elements of the ring $R$ and two elements $x, y \in R$ are adjacent iff $x+y \in Z(R)$. It is easy to show that in the case when $Z(R)$ is an ideal of $R$, one has the same properties as in the case of commutative rings.

Let us now suppose that $R$ is an arbitrary commutative ring and $M_{n}(R)$ is the ring of square matrices of order $n \geqslant 2$ over the ring $R$. It is known that in this case we have $A \in Z\left(M_{n}(R)\right)$ if and only if $\operatorname{det}(A) \in Z(R)\left[\mathbf{9}\right.$, so $Z_{L}\left(M_{n}(R)\right)=$ $Z_{R}\left(M_{n}(R)\right)=Z\left(M_{n}(R)\right)$. Of course, this set is not an ideal of $M_{n}(R)$. Let $A, B \in M_{n}(R)$ be arbitrary matrices. Then there exists a matrix $C \in M_{n}(R)$ such that $A-C-B$ is a path in $T\left(\Gamma\left(M_{n}(R)\right)\right)$. Namely, for $A=\left[A_{\downarrow 1}, \ldots, A_{\downarrow n}\right]$, $B=\left[B_{\downarrow 1}, \ldots, B_{\downarrow n}\right]\left(A_{\downarrow j}\right.$ stands for the $j$ th column of the matrix $\left.A\right)$ we choose $C=\left[-A_{\downarrow 1},-B_{\downarrow 2}, 0, \ldots, 0\right]$. It is clear that $(A+C)_{\downarrow 1}=0,(C+B)_{\downarrow 2}=0$. So, $A+C, C+B \in Z\left(M_{n}(R)\right)$. Therefore we have the following theorem.

THEOREM 3.7. Let $R$ be a commutative ring. The total graph $T\left(\Gamma\left(M_{n}(R)\right)\right)$ is connected and $\operatorname{diam}\left(T\left(\Gamma\left(M_{n}(R)\right)\right)\right)=2$.

Acknowledgments. The authors would like to thank the anonymous referee for his/her helpful comments that have improved the presentation of results in this article. 


\section{References}

1. D. F. Anderson, A. Badawi, The total graph of a commutative ring, J. Algebra 320 (2008), 2706-2719.

2. D. F. Anderson, P.S. Livingston, The zero-divisor graph of a commutative ring, J. Algebra 217 (1999), 434-447.

3. D. F. Anderson, S. B. Mulay, On the diameter and girth of a zero-divisor graph, J. Pure Appl. Algebra 210 (2007), 543-550.

4. D. D. Anderson, M. Winders, Idealization of a module, J. Comm. Algebra 1/1 (2009), 3-56.

5. M. Axtel, J. Coykendall, J. Stickles, Zero-divisor graphs of polynomials and power series over commutative rings, Comm. Algebra 6 (2005), 2043-2050.

6. M. Axtel, J. Stickles, Zero-divisor graphs of idealizations, J. Pure Appl. Algebra 204 (2006), 235-243.

7. I. Beck, Coloring of commutative rings, J. Algebra 116 (1988), 208-226.

8. I. Božić, Z. Petrović, Zero-divisor graphs of matrices over commutative rings, Comm. Algebra 37 (2009), 1186-1192.

9. W. Brown, Matrices Over Commutative Rings, Marcel Dekker, New York, 1993.

10. D. E. Fields, Zero-divisors and nilpotents in power series rings, Proc. Amer. Math. Soc. 27 (1971), 427-433.

11. R. Gilmer, A. Grams, T. Parker, Zero divisors in power series rings, J. Reine Angew. Math. 278/279 (1975), 145-164.

12. J. Huckaba, J. Keller, Annihilation of ideals in commutative rings, Pacific J. Math. 83 (1979), 375-379.

13. J. Huckaba, Commutative Rings with Zero Divisors, Monographs and Textbooks in Pure and Applied Mathematics 117, Marcel Dekker, New York, 1988.

14. I. Kaplansky, Commutative Rings, Revised Edition, University of Chicago Press, Chicago, 1974.

15. T. G. Lucas, The diameter of a zero-divisor graph, J. Algebra 301 (2006), 174-193.

16. S.P. Redmond, Central sets and radii of the zero-divisor graphs of commutative rings, Comm. Algebra 34 (2006), 2389-2401.

17. S. P. Redmond, On zero-divisor graphs of small finite commutative rings, Discrete Math. $\mathbf{3 0 7}$ (2007), 1155-1166.

18. S. P. Redmond, The zero-divisor graph of a non-commutative ring, Internat. J. Commutative Rings 1(4) (2002), 203-211.

Faculty of Civil Engineering

(Received 1504 2010)

University of Belgrade

(Revised 0702 2011)

11000 Belgrade

Serbia

pucanovic@grf.bg.ac.rs

Faculty of Mathematics

University of Belgrade

11000 Belgrade

Serbia

zoranp@math.rs 